\title{
EDITORIAL
}

\section{Preface: Centennial Physics at Peking University}

\author{
Xin-Cheng Xie \\ School of Physics, Peking University, Beijing 100871, China \\ E-mail:xcxie@pku.edu.cn,xincheng.xie@okstate.edu \\ Received September 2, 2013
}

The Year 2013 is probably as much remarkable for the development of modern physical science in China as it is for School of Physics at Peking University. One hundred years ago, the physics division was born in Peking University, as the very first physics higher education unit having appeared in modern national universities of China. In the first one hundred years, the School has made distinguished contributions to the nation and to the world in both education and academia. As we celebrate the birthday of its first century and set our sights on the next, it is my great pleasure to invite you to this special issue of Frontier of Physics on "Centennial Physics at Peking University".

In 1913, the "WuLi Men" (Physics Division) was established at Peking University, and was later renamed the Department of Physics in 1919. With the reorganization of the Chinese system of higher education in 1952, the new Physics Department of Peking University was created from the merger of the physics departments of Peking University, Tsinghua University and Yenching University. This became the premier center for physics in China. The School of Physics was established in 2001, and includes not only the traditional fields of study in physics, but also related physical sciences. Throughout its history, the School has educated and hosted many prominent physicists, including figures such as Ta-You Wu, C. N. Yang, T. D. Lee, and Kun Huang.

Today, the School of Physics includes Physics, Astronomy, Atmospheric \& Oceanic Sciences, and Nuclear Science \& Technology. Research is devoted not only to the frontiers of fundamental physics but also to the innovation of advanced technology. Major research fields include high energy physics, astrophysics and cosmology, radioactive nuclear physics, high energy-density physics, key technologies for advanced light sources and particle beams, the interaction of particle beams with materials, mesoscopic semiconductor light emission and laser physics, ultrafast physics, optical properties of artificial microstructures and mesoscopic devices, electro-magnetic properties of mesoscopic functional systems, mesoscopic theory and material computation, high-temperature superconductivity physics and devices, nano-material and devices, near-field optics, quantum materials and quantum manipulation, soft condensed matter physics, biophysics, medical physics and imaging, atmospheric physics and the environment, meteorology and climate change, physical oceanography, and many others. The School consists of eleven divisions and seven related research institutes as follows, especially in which Daniel Chee Tsui Laboratory at Peking University was established in 2012, as Prof. Tsui, 1988 Nobel Prize Laureate, became a member of the School.

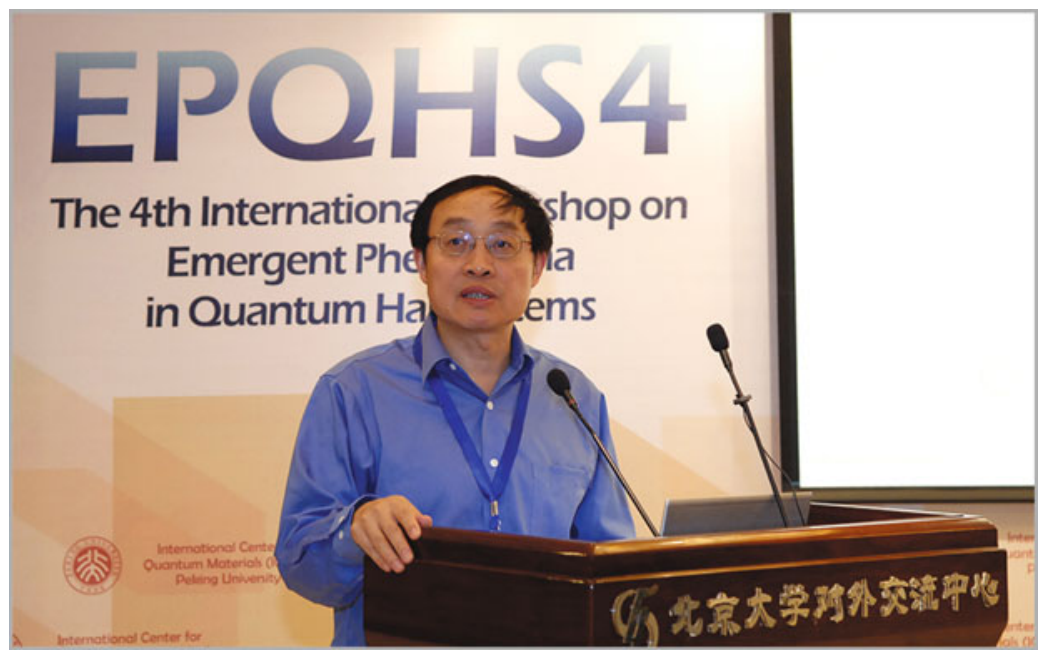


$\diamond$ Institute of Theoretical Physics

$\diamond$ Institute of Condensed Matter and Material Physics

$\diamond$ Institute of Modern Optics

$\diamond$ Institute of Heavy Ion Physics

$\diamond$ Institute of Plasma Physics and Fusion Study

$\diamond$ Department of Technical Physics

$\diamond$ Department of Astronomy

$\diamond$ Department of Atmospheric and Oceanic Sciences

$\diamond$ Teaching Center for General Physics

$\diamond$ Teaching Center for Experimental Physics

$\diamond$ Electron Microscopy Laboratory

$\diamond$ State Key Laboratory for Artificial Microstructure and Mesoscopic Physics

$\diamond \quad$ State Key Laboratory of Nuclear Physics and Technology

$\diamond$ Beijing Key Laboratory of Medical Physics and Engineering

$\diamond$ Center for High Energy Physics

$\diamond$ International Center for Quantum Materials (includes Daniel Chee Tsui Laboratory)

$\diamond$ Kavli Institute for Astronomy and Astrophysics

$\diamond$ Institute of Nuclear Science \& Technology

The School plays a leading role in planning and executing regional, national, and international scientific research programs. In the past five years, the School has more than 300 on-going and completed research projects, including 10 national basic research programs ("973" Projects), 3 national high technology research and development programs ("863" Projects) and more than 30 key projects of the National Natural Science Foundation of China (NSFC). Research funding in the School has progressively increased in recent years. Scholars in the School were awarded national and international Prizes, such as Ho Leung Ho Lee prize, National Science \& Technology Progress Awards, etc.

I am pleased to see that the selected articles in this issue, including 7 review articles and 4 research articles, cover the wide range of topics that reflects the impressive breadth of the fields in School of Physics at Peking University. In the field of optics, a summary of manipulating and utilizing the coupling between optical and mechanical degrees of freedom is provided (Hu et al., "Optomechanical sensing with on-chip microcavities"). At the nanoscale, the electronic transport properties shown in recent experiments on low-dimensional topological insulators and superconductors are summarized (Xing et al., "Electronic transport properties of topological insulator films and low dimensional superconductors"), while the strain effect is examined on semiconductors (Fu et al., "Modifying optical properties of ZnO nanowires via strain gradient"). Theoretical reviews on the particle physics level are presented on physics beyond the standard model (Hu et al., "On physics beyond standard model") and production representation of partial wave scattering amplitudes (Zheng, "Production representation of partial wave scattering amplitudes and the $f_{0}(600)$ particle"). Progress in nuclear physics is illustrated not only in the advancement of particle detection technologies ( $\mathrm{Li}$ et al., "Recent progress in subatomic particle detection technology") but also in the establishment and application of laser driven particle accelerator (Yan et al., "Recent progress of laser driven particle acceleration at Peking Univeristy") and electrostatic accelerators (Liu et al., "Frontier applications of electrostatic accelerators"). Articles in the contexts of plasma physics (Wang et al., "Low frequency Whistler waves excited in fast magnetic reconnection processes"), astronomy ( $\mathrm{Li}$, "Photon diffusion in a relativistically expanding sphere"), and atmospheric \& oceanic sciences (Wen et al., The extremely cold 2009-2010 winter and its relationship with the Arctic oscillation") are also featured in the issue.

I hope that this Special Issue of Frontier of Physics, which catches a glimpse at the current development of physics research in Peking University in celebrating the centenary of the School, will also set up a new landmark for more excellent papers from Peking University as well as from China in the future.

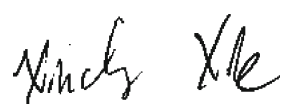

Dean, School of Physics, Peking University 